\title{
LA MORFOLOGÍA DERIVATIVA NOMINAL EN EL ZAPOTECO DE SAN PEDRO MIXTEPEC, OAXACA
}

\author{
Nominal Derivational Morphology in San Pedro Mixtepec Zapotec, Oaxaca
}

\author{
Pafnuncio Antonio Ramos*
}

\begin{abstract}
RESUMEN
En este artículo se describe la morfología derivativa nominal del idioma zapoteco hablado en la comunidad de San Pedro Mixtepec, Oaxaca. En esta lengua existen cuatro morfemas utilizados para derivar sustantivos. El primero es el morfema nominalizador $w$ - que se prefija a bases verbales o nominales para la derivación de sustantivos. El segundo es el morfema gièl-, el cual se prefija a bases adjetivales, verbales o nominales para derivar también nuevos nominales, incluso, en ciertos casos, se adhiere a verbos ya nominalizados. El tercero es el morfema derivativo $n g w$-, con sus alomorfos $\{n g w-\},\{n k w-\},\{n g-\}$ y $\{n-\}$, el cual, al agregarse a bases verbales, deriva nombres de agentes y, al prefijarse a topónimos, deriva gentilicios. El último es el morfema derivativo para nombres de animales $m$ - con sus alomorfos $\{m-\}$ y $\{n$ - $\}$. Este último sistema representa la reminiscencia de la gramaticalización de, por lo menos, dos elementos léxicos con orígenes del protozapotecano y protozoqueano, los cuales han pasado por varios procesos fonológicos hasta convertirse en el prefijo que actualmente ocurre al inicio de un gran número de nombres de animales.
\end{abstract}

Palabras clave: Morfología, derivación, nombres, prefijos, zapoteco.

\begin{abstract}
In this paper, I describe nominal derivational morphology in the Zapotec language spoken in the community of San Pedro Mixtepec, Oaxaca. In this language, there are four morphemes used to derive nouns. The first one is the $w$ - morpheme. This prefix is used in verbal or nominal roots to derive nouns. The second one is the prefix giel-. This is a nominalizing morpheme, which occurs with adjectival, verbal or nominal roots to derive new nouns, and there are even instances in which this morpheme is prefixed to already nominalized verbs. The third one is the morpheme $n g w$-, with its allomorphs $\{n g w-\},\{n k w-\},\{n g-\}$ and $\{n-\}$. This morpheme attaches to verbal roots to derive agent nouns. However, when it is attached to place names, it derives demonyms. The last one is a morpheme that occurs with animal names $m$ - with its allomorphs $\{m-\}$ and $\{n-\}$. This last system represents what has remained of the grammaticalization of at least two lexical elements originating in the protozapotecan and protozoquean linguistic families, which have gone through several phonological processes until becoming prefixes that occur at the beginning of animal names.
\end{abstract}

Key Words: Morphology, derivation, nouns, prefixes, Zapotec.

*Universidad de Sonora. Doctor en Lingüística Indoamericana por el Centro de Investigaciones y Estudios Superiores en Antropología Social (CIESAS). México.

Correo electrónico: paf217@hotmail.com

Recepción: 20/03/2019.

Aprobación: 19/07/2019. 


\section{Introducción ${ }^{1}$}

En el presente artículo se estudia la morfología derivativa nominal del zapoteco de San Pedro Mixtepec (a partir de ahora ZSPM). Los temas que aquí se tratan, principalmente, abordan los mecanismos morfológicos que la lengua emplea para la derivación de nominales.

La morfología derivativa es el mecanismo que los idiomas emplean para crear palabras para nuevos conceptos (Haspelmath, 2002, p. 68; Bauer, 2003, p. 91; Lieber, 2017, p. 2). Las categorías léxicas que generalmente participan en la derivación son nombres, verbos, adjetivos y adverbios. Al agregar material morfológico derivativo a una base léxica libre, pueden ocurrir cambios de tipo semántico o cambios de clase léxica.

Por su parte, Aronof y Fudeman (2011) proponen tres características principales para determinar los procesos de derivación: i) cambio de significado léxico o de categoría, ii) tendencia a ocurrir cerca de la raíz y iii) presencia en el lexicón. Entre los cambios de clase léxica más comunes tenemos que un verbo puede cambiar a nombre, un nombre, a adjetivo, o un adjetivo, a un adverbio (Genetti, 2014, p. 90). Según Haspelmath (2002), la nominalización es el proceso derivativo más frecuente, le sigue la verbalización, después, la adjetivación y, finalmente, la adverbialización (p. 69). Así, los medios formales por los cuales ocurre la derivación son la afijación, la reduplicación o algún tipo de modificación interna (Lieber, 2017, p. 2).

En el zapoteco de San Pedro Mixtepec existen cuatro morfemas derivativos bases que se utilizan para formar nombres: el primero es el morfema nominalizador $w$-; el segundo, el morfema nominalizador gièl-; el tercero es el morfema derivativo $n g w-$, con sus alomorfos $\{n k w-\},\{n g-\}$ y $\{n-\}$, finalmente, el cuarto morfema es el nasal bilabial $m$ - con su único alomorfo $\{n-\}$.

\footnotetext{
${ }^{1}$ Agradezco a mis colaboradores zapotecos: Alberto Antonio Méndez (†2019) y Manuel Antonio Cruz por los datos que me compartieron para esta investigación. Al Consejo Nacional de Ciencia y Tecnología por el soporte económico que me otorgó mediante la beca Estancias Posdoctorales al Extranjero 2017-2018. A la Universidad de California, Santa Bárbara, por aceptarme como posdoctorante en su institución. Sin estos apoyos no hubiera sido posible esta investigación.
} 
De los cuatro morfemas derivativos mencionados, dos de ellos, el morfema $n g w$ - y $n$-, ya se habían descrito en un estudio sobre la categorización nominal en el ZSPM (Antonio-Ramos, 2015, p. 459). En dicho estudio, el morfema $n g w$ - se describe como prefijo de la clase de sustantivos asignados a humanos y el morfema $n$ - como prefijo asignado a la clase de sustantivos referidos a los animales. No obstante, los dos morfemas restantes $w$ - y gièl-, no se habían reportado, ni en estudios de la lengua zapoteca en general, ni en investigaciones propias del ZSPM. Es necesario comentar que estos cuatro morfemas representan una parte importante del sistema de derivación nominal en el ZSPM.

Para los procesos de derivación nominal, se observa que cada prefijo nominalizador requiere, obligatoriamente, de una base léxica. Dichas bases léxicas pueden ser del tipo nominal, verbal, adjetival o incluso bases ya nominalizadas. El morfema $w$-, por ejemplo, emplea dos tipos de bases: las bases verbales simples y las complejas. Estas últimas se forman de un verbo más un sustantivo. El morfema gièl- emplea bases adjetivales, verbales, nominales y bases ya nominalizadas. Se observan también bases que muestran opacidad semántica. Estos últimos son raíces que ya no tienen un significado propio al encontrarse en su forma simple.

A continuación, se presentan de manera breve las generalidades del ZSPM. Posteriormente, se describe el análisis de la derivación prefijal del ZSPM, el cual se compone de cuatro subapartados: la nominalización con el morfema derivativo w-, la nominalización con el morfema derivativo gièl-, la derivación de nombres de agentes y gentilicios y la nominalización de nombres de animales. Finalmente, se muestran las conclusiones. 


\section{Generalidades del ZSPM}

\section{La lengua zapoteca y la comunidad de estudio}

El zapoteco de San Pedro Mixtepec es una de las variedades dialectales del idioma zapoteco Cisyautepequeño (Smith-Stark, 2007; Beam de Azcona, 2018). Este idioma junto con el zapoteco de los valles centrales y del istmo conforma el grupo lingüístico zapoteco central. El zapoteco central, el miahuateco y el zapoteco del norte conforman el grupo zapoteco medular. Y estos junto con el solteco y el zapoteco occidental conforman la macrolengua zapoteca.

San Pedro Mixtepec es una comunidad rural ubicada en las montañas de la región Sierra Sur del estado de Oaxaca, a doscientos kilómetros de distancia de la ciudad capital, aproximadamente. De acuerdo con los resultados del censo de población, cuenta con mil setenta y siete personas, la mayoría hablante de la lengua zapoteca (Instituto Nacional de Estadística y Geografía, 2015).

\subsection{Características gramaticales básicas del zapoteco de San Pedro Mixtepec}

El sistema fonológico del ZSPM está compuesto de veinticuatro fonemas consonánticos y dos aproximantes (Antonio-Ramos, 2015, pp. 88-90). De estos veintiséis elementos se derivan las veintiséis grafías, las cuales constituyen el sistema ortográfico del ZSPM (ver tabla 1). Dicha ortografía práctica es retomada para ejemplificar los datos en este artículo. ${ }^{2}$

\footnotetext{
${ }^{2}$ En el ZSPM, existen algunos fonemas consonánticos no muy comunes en otros idiomas, su forma ortográfica y sus correspondencias fonológicas son las siguientes: $\langle\mathrm{kw}\rangle=/ \widehat{\mathrm{kw}} /,\langle\mathrm{gw}\rangle=/ \widehat{\mathrm{gw}} /,\langle\mathrm{ts}\rangle=/ \widehat{\mathrm{ts}} /$,

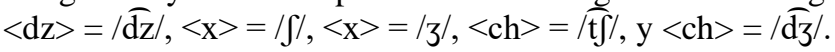


Tabla 1. Inventario de consonantes ortográficas del ZSPM

\begin{tabular}{|c|c|c|c|c|c|c|c|}
\hline & Oclusivas & Fricativas & Africadas & Nasales & Laterales & Vibrantes & $\begin{array}{l}\text { Aproxi } \\
\text { mantes }\end{array}$ \\
\hline $\begin{array}{l}\text { Grafías } \\
\text { fortis }\end{array}$ & $\langle\mathrm{p}, \mathrm{t}, \mathrm{k}, \mathrm{kw}\rangle$ & $\langle\mathrm{f}, \mathrm{s}, \mathrm{x}\rangle$ & $\langle\mathrm{ts}, \mathrm{ch}\rangle$ & $<\mathrm{m}, \mathrm{n}>$ & $<1>$ & $\langle\mathrm{r}\rangle$ & -- -- \\
\hline Grafías lenis & $\langle\mathrm{b}, \mathrm{d}, \mathrm{g}, \mathrm{gw}\rangle$ & $\langle--\mathrm{Z}, \underline{\mathrm{X}}\rangle$ & $<\mathrm{dz}, \underline{\mathrm{ch}}>$ & $\langle--\underline{n}\rangle$ & $\langle\underline{1>}$ & $\langle\mathrm{r}\rangle$ & $\langle\mathrm{y}, \mathrm{w}\rangle$ \\
\hline
\end{tabular}

Fuente: Elaboración propia.

El sistema vocálico del ZSPM se constituye de seis timbres vocálicos (a, e, i, o, u y ë) y un contraste entre dos tipos de voz: la modal y la no modal. El contraste entre estos dos tipos de voz posibilita la existencia de dos clases de vocales fonológicas: las vocales modales y las no modales. De estos dos juegos fonológicos de vocales, se deriva el inventario ortográfico (tabla 2) y es el que se utiliza para la escritura de los datos en este trabajo. ${ }^{3}$

Tabla 2. Inventario de vocales ortográficas del ZSPM

\begin{tabular}{|c|c|c|c|}
\hline & Anterior & Media & Posterior \\
\hline Altas & $<\mathrm{i}, \mathrm{i}^{\prime}>$ & & $<\mathrm{u}, \mathrm{u}^{\prime}>$ \\
\hline Media & $<\mathrm{e}, \mathrm{e}^{\prime}>$ & & $<0, \mathrm{o}^{\prime}>$ \\
\hline Media baja & $<\ddot{e}, \ddot{e} ’>$ & & \\
\hline Baja & & $<\mathrm{a}, \mathrm{a}^{\prime}>$ & \\
\hline
\end{tabular}

Fuente: Elaboración propia.

El ZSPM es una lengua tonal con un inventario de cinco tonemas. Los cuatro primeros son tonos léxicos y el último es producto de procesos de sandhis tonales (véase tabla 3).

Tabla 3. Inventario tonal del ZSPM

\begin{tabular}{lll}
\hline Tipos tonales & Tonos & Forma ortográfica \\
\hline Tonos de nivel & Tono bajo & $<^{\prime}>$ \\
\cline { 2 - 3 } & Tono alto & $\left.<^{\prime}\right\rangle$ \\
\hline
\end{tabular}

\footnotetext{
${ }^{3}$ Las formas ortográficas de las vocales no modales y sus correspondencias fonológicas son $<$ a' $>=/ \mathrm{a} P /,<\ddot{\mathrm{e}}>$ $=/ \mathfrak{Q} \mathrm{P} /,<\mathrm{e}^{\prime}>=/ \mathrm{e} \mathrm{P} /, \mathrm{i}^{\prime}>=/ \mathrm{i} \mathrm{P} /,<_{\mathrm{O}}{ }^{\prime}>=/ \mathrm{o} \mathrm{P} /, \mathrm{y}$ la $<\mathrm{u}^{\prime}>=/ \mathrm{u} \mathrm{P} /$.
} 


\begin{tabular}{lll}
\hline Tonos de contorno & Tono bajo ascendente & $\left\langle{ }^{`}\right\rangle$ \\
\cline { 2 - 3 } & Tono alto ascendente & $\langle "\rangle$ \\
\cline { 2 - 3 } & Tono descendente & $\left\langle{ }^{\wedge}\right\rangle$ \\
\hline
\end{tabular}

Fuente: Elaboración propia.

El ZSPM es una lengua de verbo inicial. En construcciones intransitivas, el orden es verbo, sujeto como en (1a); en construcciones monotransitivas, el orden es verbo, agente, paciente como en (1b); en construcciones ditransitivas de objeto oblicuo, el orden es verbo, agente, tema, recipiente como en (1c); mientras que en construcciones ditransitivas directas, el orden es flexible como en (1d) y (1e).

(1)a. b-ì'n chěnch ${ }^{4}$

CPL-llorar Crescencia

'Crescencia lloró'.

b. b-dě'd lën gőn

$(\mathrm{tx})$

CPL-dar Elena limosna

'Elena dio la limosna'.

c. b-găd xěy nèx ló rrós $\quad(t \mathrm{t})$

CPL-regalar señor fruta SR: cara Rosa

'El señor le regaló frutas a Rosa'.

d. ptìbě̆d chiìkw mèdwinn lár

(tx)

\footnotetext{
${ }^{4}$ Las abreviaturas que se utilizan en este artículo son $1 \mathrm{SG}=$ primera persona singular, $2 \mathrm{SG}=$ segunda persona singular, $3 \mathrm{ANIM}=$ tercera persona animal, $3 \mathrm{INAN}=$ tercera persona inanimado, $\mathrm{CPL}=$ completivo, $\mathrm{DEM}=$ demostrativo, INAN $=$ inanimado, $\mathrm{NOM}=$ nominalizador, $\mathrm{SSS}=$ sin semántica individual, $\mathrm{SR}=$ sustantivo relacional, $\mathrm{TX}=\mathrm{de}$ texto, $\mathrm{ZSPM}=$ zapoteco de San Pedro Mixtepec.
} 
CPL-envolver Francisco niño trapo

'Francisco envolvió al niño con una trapo'.

e. ptìbë̀d chìkw lár mèdwǐn

CPL-envolver Francisco trapo niño

'Francisco envolvió al niño con un trapo'.

El sistema de alineamiento en construcciones monotransitivas es nominativo-acusativo y se determina por el orden de los constituyentes. Por tanto, la posición nominativa se encuentra inmediatamente después del verbo como en (2a), y posterior a él se encuentra la del acusativo como en $(2 b)$.

(2)a. b-dín juän màrí

CPL-pegar Juan María

'Juan le pegó a María'.

b. b-dín màrí Juán

CPL-pegar María Juan

'María le pegó a Juan'.

En una construcción ditransitiva como en (3a), es claro que el objeto directo está después del sujeto e inmediatamente después del objeto directo se coloca el objeto indirecto. Sin embargo, cuando es necesario darle mayor prominencia al objeto indirecto, este se focaliza colocándose después del elemento verbal como en (3b). Estas dos posibilidades de orden entre los objetos permiten que el 
alineamiento sea del tipo neutro. Nótese el cambio tonal que ocurre en la palabra bàygiét al estar en la posición final de la construcción y bàygièt al encontrarse en la posición intercláusal. ${ }^{5}$

$\begin{array}{llll}\text { (3)a. p-tibĕ̈d } & \text { màrí } & \text { gìxtíl bàygiét } & \\ \text { CPL-envolver mari } & \text { pan } & \text { servilleta }\end{array}$

'María envolvió el pan con la servilleta'.

b. p-tìbĕ̈d màrí bàygièt gìxtíl $\quad(\mathrm{tx})$

CPL-envolver mari servilleta pan

'María envolvió el pan con la servilleta'.

En construcciones ditransitivas con oblicuos, el alineamiento es del tipo secundativo (Malchukov, Haspelmath y Bernard, 2010, p. 4). El paciente y el tema no necesitan de ninguna marca para introducirse, mientras que el recipiente se integra con el sustantivo relacional lo 'cara'. Véase (4a) y (4b) como ejemplos.

(4)a. b-nëdz nà dmí

CPL-dar 1SG dinero

'Yo di dinero'.

b. b-děd nà dmï ló glórià

$(\mathrm{tx})$

CPL-dar 1SG dinero SR:cara Gloria

'Yo le di dinero a Gloria'.

\footnotetext{
${ }^{5}$ Los cambios tonales que aquí se observan no tienen implicaciones sintácticas. Estos son procesos de sandhis tonales que caracterizan a los sustantivos inanimados (véase Antonio-Ramos, 2015).
} 


\section{Análisis de la derivación prefijal del ZSPM}

El ZSPM es una lengua con cuatro morfemas derivativos. El primero es el morfema nominalizador w-, el cual se utiliza como prefijo de una raíz verbal para derivar nombres de eventos. El segundo es el morfema nominalizador gièl-, utilizado como prefijo en nominales simples para derivar nombres abstractos. El tercero es el morfema derivativo $n g w$-, el cual origina nombres de agentes cuando las bases son verbales, y, gentilicios cuando las bases son topónimos. El cuarto es el morfema derivativo nasal bilabial $m$-, conocido como morfema de clase nominal. Este último morfema se ha gramaticalizado de elementos léxicos del protozapotecano y protozoqueano; actualmente, se comporta como prefijo y se coloca en los inicios de nombres de animales. Además, hay una combinación de los morfemas gièl- + w- que se utiliza para formar nombres con una semántica abstracta (véase tabla 4).

Tabla 4. Inventario de morfemas derivativos prefijales

\begin{tabular}{ll}
\hline Función derivativa léxica & Prefijos \\
\hline Nominalización de verbos & w- + verbo \\
& w- + verbo + nombre \\
\hline Nominalización de eventos & gièl- + adjetivo \\
& gièl- + w + verbo \\
\hline Nombres de agentes & ngw-, nkw-, ng-, n- \\
\hline Gentilicios & ngw-, nkw-, ng-, n- \\
\hline Nombres de animales & m-, n- \\
\hline
\end{tabular}

Fuente: Elaboración propia.

\subsection{Nominalización con el morfema derivativo $w$ -}

El primer proceso de derivación que aquí se presenta es la nominalización con el morfema $w$-. Existen dos subtipos de nominalizaciones con dicho prefijo. El primero se forma del nominalizador w- más una raíz verbal, mientras que el segundo subtipo se constituye del mismo nominalizador $w$ - 
más una raíz compleja compuesta de una base verbal más una base nominal.

En (5) se presenta el primer subtipo de nominalizaciones con el nominalizador $w$ - más una base verbal. En la primera columna, se muestran las formas nominalizadas en donde se segmenta la raíz verbal y el morfema nominalizador, y en la segunda columna se ubican las raíces verbales flexionadas en incompletivo $/ r-/ .{ }^{6}$ Todos los verbos de la lista de (5) inician con fonemas consonánticos. Estos tienen un comportamiento regular al nominalizarse, ya que mantienen intacta sus raíces. Además, sus raíces inician con vocales, las cuales emplean formas supletivas al cambiar de una categoría nominal a una verbal, sin embargo, las dos formas emplean el prefijo derivativo. Véase los ejemplos en (6).

(5)Nominalización con el prefijo derivativo $w$ - más raíz verbal
a. w-gìt
'el juego o el juguete'
r-gìt 'jugar'
b. w-kièts
'el entierro'
r-kiè'ts 'enterrar'
c. W-tò
'la venta'
r-tò 'vender'
d. w-sè'd
'el aprendizaje'
r-sè'd 'aprender'
e. w-dì'dz
'la plática'
r-dì'dz 'platicar'
f. w-tiǐb
'la enrollada'
r-tǐb 'enrollar'

(6) Nominalización con el prefijo derivativo $w$ - más raíz verbal
a. w-i'l
'el canto'
r-ò'l 'cantar'
b. w-ìn
'el llanto'
r-ò'n 'llorar'

\footnotetext{
${ }^{6}$ El morfema del incompletivo es la forma más regular en la flexión verbal y es la que permite segmentar con claridad la raíz nominal y el prefijo flexivo.
} 


$\begin{array}{llll}\text { c. w-jě' } & \text { 'el baile' } & \text { r-iě' } & \text { 'bailar' } \\ \text { d. w-è't } & \text { 'la molienda' } & \text { r-òt } & \text { 'moler' } \\ \text { e. w-ágw } & \text { 'la comida' } & \text { r-áw } & \text { 'comer' }\end{array}$

En (7) se presenta un tipo de nominalización compleja, que se forma del morfema nominalizador $w$ más una raíz verbal y un sustantivo inanimado. Estos nominales derivados complejos no son formas flexivas verbales, esto es, porque carecen de los morfemas del TAM. Asimismo, estos sustantivos no tienen una equivalencia léxica en español. La mayoría de estas nominalizaciones cuentan tanto con el significado de sus raíces verbales como de los significados de sus raíces nominales. Sin embargo, hay casos en los cuales los nominales ya no son reconocidos en la variante de estudio, como làdz en (7b) y (7c). La única manera de conocer el significado de estos sustantivos es rastrearlos en las otras variedades zapotecas.

(7)Nominalización con el prefijo derivativo $w$ - más una raíz verbal y una raíz nominal
a. r-tsè
'cerrar'
W-tsè-nìs
'la cerrada de agua'
nìs 'agua'
b. rsó
'empezar'
w-sò-làdz
'el recordatorio'
làdz $\quad$ 'corazón'
c. rzó
'pararse'
w-zò-làdz
'el descanso'
làdz 'corazón'
d. rlă'gw
'aparearse'
w-lăgw-lsâ
'el apareamiento'

\footnotetext{
${ }^{7}$ Làdz es cognado con làch y es la palabra que significa 'semilla' o 'corazón' para el zapoteco de San Juan Guiviní.
} 
lsà 'parientes'

e. rdǐ’b 'enrollar' w-dì̀-bĕ́d 'la enrollada'

bë̀d 'revuelta'

f. r-kă'dz 'machucar' w-kădz-gí’b 'el labrado de metales'

gì'b 'metal'

g. r-xìdù' 'amarrar' w-xìdù-gìx $\quad$ 'la amarrada de zacate'

gìx $\quad$ 'zacate'

\subsection{Nominalización con el morfema derivativo gièl-}

El segundo tipo de nominalización es el que surge del prefijo silábico gièl-. Estas nominalizaciones se clasifican en cuatro subtipos: i) nominalizaciones constituidas de un morfema derivativo prefijal gièl- más un adjetivo, ii) nominalizaciones que se forman del prefijo derivativo más una raíz verbal, iii) nominalizaciones que se originan del prefijo derivativo más una raíz nominal, y iv) nominalizaciones que se forman del prefijo derivativo gièl- más una palabra que ya se ha nominalizado con el prefijo derivativo $w$ -

En (8) se presentan los ejemplos del tipo i). Todos los adjetivos en sus formas aisladas tienen sus significados propios, no obstante, al nominalizarse, cambian su semántica. Nótese que todos los ejemplos inician con un fonema nasal alveolar $/ n /$. En la mayoría de los casos, no se observan cambios tonales al pasar de la categoría de adjetivo a nombre. Sin embargo, hay un caso que no

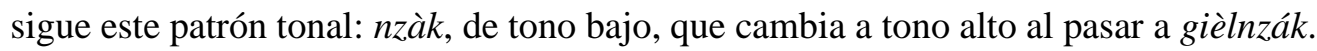


(8)Prefijo nominalizador giel- más adjetivo
a. ndzě’b 'malo' gièl-ndzě’b 'el peligro'
b. ndzín 'inquieto' gièl-ndzín 'la inquietud'
c. ndzăb 'feo' gièl-ndzăb 'la fealdad'
d. ngì 'bueno' gièl-ngì 'la bondad'
e. nzàk 'sagrado' gièl-nzák 'la fortuna'
f. ngiěs 'travieso' gièl-ngiěs 'la travesura'
g. ndä'n 'saludable' gièl-ndä'n 'la salud'
h. ngăn 'difícil' gièl-ngăn 'la dificultad'
i. nsès 'veloz' gièl-nsès 'la habilidad'

El subtipo de nombres derivados ii) se ejemplifica en (9). Estas raíces verbales, al ser prefijadas con el morfema gièl-, se convierten en sustantivos. Estas raíces verbales jamás pueden encontrarse de forma aislada.

(9) Nominalización con el prefijo gièl- más una forma verbal
a. Gièl-nề
'la aflicción'
b. Gièl-mbän
'la vida'
c. Gièl-mbăn
'la tristeza'
d. Gièl-gùt
'la muerte' 

e. Gièl-rriën
'el cerebro'
f. Gièl-kièw
'la obscuridad'
g. Gièl-tő
'la vergüenza'

Para el subtipo de nominalizaciones iii) únicamente se registran dos casos. El primero es tsiěl 'conyugue' y el segundo es $z \grave{l}$ 'pegamento'.

(10) Nominalización con el prefijo nominalizador gièl- más un sustantivo
a. tsiě'l
'concubino'
gièl-tsiè'1
'el casamiento'
b. ž
'pegamento'
gièl-žr
'la maldad'

El último subtipo de nominalizaciones iv) se ejemplifica en (11). Las raíces verbales que participan en este tipo de palabras derivativas en ningún contexto pueden realizarse de manera aislada. Esto significa que siempre se realizarán con otras marcas morfológicas.

(11) Nominalización compleja con el prefijo gièl- más el nominalizador $w$ -
a. Gièl-w-là'gw 'el apareamiento'
b. Gièl-w-ágw 'la comida'
c. Gièl-w-xîn 'la oscuridad'
d. Gièl-w-lě 'la felicidad'
e. Gièl-w-lë’ 'el enojo'
f. Gièl-w-ěgw 'la indiscreción'

${ }^{8} Z \check{l}$ es una palabra zapoteca que se refiere a un tipo de pegamento que las personas elaboraban para atrapar
aves. 


\subsection{Derivación de nombres de agentes y gentilicios}

El tercer tipo de derivación nominal ocurre con el prefijo derivativo nasal alveolar $/ n-/$ y sus alomorfos $\{n-\},\{n g-\},\{n g w-\}$ y $\{n k w-\}$. Este proceso derivativo se organiza en dos subtipos. El primer subtipo genera nombres de agente, mientras que el segundo, gentilicios. Los nombres de agentes se forman de la intervención del prefijo derivativo más un verbo de acción. Por su parte, en la formación de los gentilicios intervienen el morfema derivativo más un topónimo.

Para el uso de los alomorfos derivativos $\{n-\},\{n g-\},\{n g w-\}$ y $\{n k w-\}$, se observa cierto condicionamiento fonológico. Este condicionamiento se relaciona con los inicios de las bases verbales y el segmento final de los alomorfos derivativos. Por ejemplo, el alomorfo base $\{n g w-\}$ se realiza antes de fonemas vocálicos y laterales lenis como en (12a) y (12b); el fonema $\{n k w$ - $\}$ ocurre antes de la consonante oclusiva alveolar / $t /$ como en (12c); el alomorfo $\{n g-\}$ se realiza antes de consonantes bilabiales lenis como en (12d); y el alomorfo nasal alveolar se produce antes del fonema velar lenis como en (12e).

(12) Nombres de agente formados de un verbo de acción más un prefijo derivativo

a. ngw-àgw

PREF:DER-comer

b. ngw-lăs

PREF:DER-adelgazar

c. nkw-tò

PREF:DER - vender

d. ng-bà'n 'el comensal'

$$
\text { 'el gentil' }
$$

$$
\text { 'el vendedor' }
$$

$$
\text { 'el ladrón' }
$$


PREF:DER-robar

e. n-gì- $\underline{x}$ '̀l 'el danzante

PREF:DER-bailar-algodón

En este mismo grupo derivativo, existen sustantivos que presentan raíces para las cuales no es posible saber a qué categoría pertenecen, pues ya no tienen un significado propio en su forma aislada, es decir, son palabras con opacidad semántica. Algunos ejemplos se presentan en (13).

(13) Sustantivos con opacidad semántica en las raíces
a. ng-òp
'la tortillera'

PREF:DER-SSI

b. ng-ǒp

'el discapacitado'

PREF:DER-SSI

c. nk-tò'

'el muerto'

PREF:DER-SSI

d. ngw-zì

'el rayo'

PREF:DER-SSI

e. ngw-zà

'el encantamiento'

PREF:DER-SSI 
Un grupo más de nombres que se construyen por procesos derivativos son los gentilicios. Para su formación, intervienen los alomorfos $\{n-\},\{n g-\},\{n g w-\}$ y $\{n k w-\}$, así como las raíces libres a las que estos se prefijan. Las raíces libres que participan en esta derivación son los topónimos, los cuales se clasifican en dos tipos: los topónimos que tiene una semántica general como en (14a), (14b) y (14c), y los topónimos específicos, que son nombres de pueblos, como los de (14d) a (14h).

(14) Gentilicios formados por procesos de derivación

a. ngw-lèts

PREF:DER-valle

b. n-gdò'

PREF:DER-serranía

c. n-gzè

PREF:DER-planicie

d. n-gdän

PREF:DER-Quioquitani

e. n-grè

PREF:DER -Quierí

f. n-gdzăp

PREF:DER-Quiechapa

g. n-gìzdò' 'vallista'

'serrano'

'istmeño'

'quioquitañero'

'quierillano'

'quiechapeño'

'miahuateco' 
PREF:DER-Miahuatlán

h. n-gìgw-lán 'quiegolanero'

PREF:der-Quiegolani

Existen topónimos como lă 'Oaxaca', zgìt 'Tenochtitlan' y muchos otros que no son compatibles con los alomorfos derivativos, ya que no pueden derivar gentilicios. Así, al agregarle el prefijo derivativo, resultan agramaticales como en (15a) y (15c). Para expresar los gentilicios con estos topónimos, se emplea la forma mén lă 'gente de Oaxaca' y mén zgít 'gente de Tenochtitlán' como en $(15 b)$ y $(15 d)$.

(15) Topónimos que no aceptan los alomorfos derivativos
a. *ngw-lă
'oaxaqueño'

PREF:DER-Oaxaca

b. mén lă 'oaxaqueño'

'persona' 'Oaxaca'

c. *ngw-zgìt 'mexica'

PREF:DER-tenochtitlán

d. mén zgít 'mexica'

‘persona' 'Tenochtitlán'

${ }^{9}$ Gentilicio utilizado para nombrar a las personas que vivieron en Tenochtitlán, una antigua ciudad de Mesoamérica. 
Los alomorfos derivativos en nombres de agentes y gentilicios se encuentran en distribución complementaria. Esta distribución se presenta con la siguiente regla fonológica contextual.

$$
\left\{\mathrm{ngw}_{-}\right\}\left\{\begin{array}{l}
\mathrm{n}- \\
\mathrm{ng}- \\
\mathrm{nk}-/ \mathrm{nkw}- \\
\mathrm{ngw}- \\
\mathrm{nkw}-
\end{array}\right\} \quad \begin{aligned}
& -\mathrm{velar} \text { lenis } / \mathrm{g} / \\
& -\mathrm{v} \mathrm{o} \_/ \mathrm{b} / \\
& -/ \mathrm{t} / \\
& -\mathrm{C} \text { lenis excepto } / \mathrm{b} /, / \mathrm{g} / \mathrm{y} \text { vocales } \\
& -/ \mathrm{k} /
\end{aligned}
$$

Regla 1. Los alomorfos derivativos para los nombres de agentes y gentilicios.

\subsection{Derivación de nombres de animales}

Existe también un grupo de palabras derivadas que se utiliza para nombrar animales. Estas palabras se forman de un prefijo derivativo nasal $/ m$-/ con sus dos alomorfos $\{m-\}$ y $\{n-\}$ más una raíz léxica. El morfema derivativo que se observa en los nombres de animales es producto de una fusión de, por lo menos, tres componentes: dos unidades léxicas y un afijo de animacidad. El primer componente es *niP 'animal', del protozapotecano; el segundo es *mน?a 'venado', del protozoqueano (Kaufman, 2016, p. 6), y el tercero es el morfema de animacidad *kwe $7^{10}$ 'aire', 'viento' o 'aliento', del protozapoteco (Kaufman, 2006, p. 15). El fonema labiovelar $k w$ de la palabra *kwe- del protozapoteco cambió a $p$ - al pasar en el zapoteco. A raíz de este cambio, el morfema kwe- se convirtió en pee- en el zapoteco colonial (De Córdoba, 1578). Esta misma forma pee- fue descrita con la semántica de 'aire', 'ánima', 'espíritu' o 'fuerza vital' (Marcus y Kent, 1978, p. 151).

\footnotetext{
${ }^{10}$ Kauman utiliza el símbolo 7 para representar la glotal.
} 
Por otro lado, los nominales *ni? ‘animal' y *mu?a 'venado' se juntaron y formaron la palabra *mmani? 'animal'; posteriormente, este último se unió al prefijo derivativo para los nombres de animales del protozapoteco $k^{w} e-$ y formó $*_{m m a-n i ?-k^{w} e-;}$ una vez juntos, se prefijaron a raíces nominales y resultaron así en nombres de animales, como *mma-ni?-kwe-fiPtso? 'tejón'. Posteriormente, la forma *mma-ni?-kwe-pasó por varios procesos fonológicos hasta convertirse en las formas fusionadas de $b$ - para el zapoteco del istmo, $m$ - para el zapoteco de Mixtepec, y $m b$ - para el zapoteco de Coatlán Loxicha. La forma prefijal $b$ - puede apreciarse en palabras como be te 'zorrillo', beedze' 'tigre', bere 'gallina' (Pickett, Black y Cerqueda, 1998, pp. 5-8). La forma mpuede apreciarse en el zapoteco de Mixtepec en palabras como m-èt 'zorrillo', m-è 'dz 'tigre' y m-èr 'guajolote' (Antonio-Ramos, 2015, pp. 126-193). Y la forma $m b$ - para el zapoteco de Coatlán Loxicha en palabras como mbith 'zorrillo', $m b i^{7} z h$ 'tigre', y mbeit 'guajolote' (Beam de Azcona, 2004, pp. 374-476). Esto demuestra la procedencia del morfema derivativo que presentan los nombres de animales.

En párrafos anteriores, se anunciaron los alomorfos derivativos nasal bilabial $\{m-\}$ y la nasal alveolar $\{n-\}$, ambos se encuentran en distribución complementaria. La nasal alveolar ocurre antes de consonantes velares, excepto en las palabras mgìd 'pulga' y mgi'n 'pájaro'. También, aparece antes de la consonante africada / $\overparen{t f /}$ que ortográficamente se representa como $\langle c h\rangle$. No obstante, la nasal bilabial ocurre en los demás contextos. Véase los ejemplos en (16) y (17).

(16) Morfema derivativo nasal alveolar a inicio de nombres de animales

a. n-gǒn 'ganado' animal-ganado

b. n-gǐd 'gallina' animal-gallina 


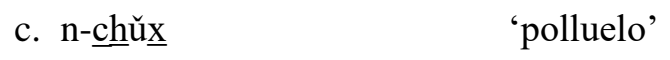

animal-polluelos

d. n-gwläz 'mosca'

animal-moscas

(17) Morfema derivativo nasal bilabial a inicio de nombres de animales

a. m-ềkw 'perro'

animal-perro

b. m-gín 'pájaro'

animal-pájaro

c. m-sì 'águila'

animal-águila

d. m-ziǒn 'coralillo'

animal-coralillo

e. m-tř't 'chinche'

animal-chinche

f. m-kí 'liendre'

animal-liendre 
Existe un grupo pequeño de sustantivos que, desde la cosmovisión occidental, son plantas; sin embargo, estos nombres zapotecos llevan el prefijo nasal $m$-, utilizado para los nombres de animales. Los ejemplos se pueden ver en (18).

(18) Sustantivos con el prefijo utilizado para los nombres de animales
a. m-ěy
'hongo'
animal-hongo
b. $m-\ddot{e}$
'frijol'
animal-frijol

No es posible afirmar que en la cosmovisión zapoteca estas plantas se consideren animales, pero se observa que para pronominalizarlas se utiliza el pronombre de tercera persona animal má; ejemplos de este tipo se presentan en (19). En contraste, otras plantas se pronominalizan con $=a$, pronombre de tercera persona inanimado, como se muestra en (20).

(19) Plantas pronominalizadas con el pronombre de tercera persona animal
a. pà
b-dziěl
lú měy
dónde CPL-encontrar 2SG hongos
¿Dónde encontraste hongos?’.
b. gìk gí yí b-dziěl nà má
SR: cabeza cerro DEM CPL-encontrar 1SG 3ANIM

'En la punta de ese cerro los encontré (hongos)'. 
(20) Plantas pronominalizadas con el pronombre de tercera persona animal

a. pà b-dziěl lù nkwàniè

dónde CPL-encontrar 2SG chipiles

¿¿Dónde encontraste chipiles?’

b. gìk gí yí bà =w

SR: cabeza cerro DEM CPL-encontrar 1SG=3INAN

'En la punta de ese cerro los encontré (chipiles)'.

En la siguiente regla fonológica, se muestran los contextos de los morfemas derivativos de los nombres de animales.

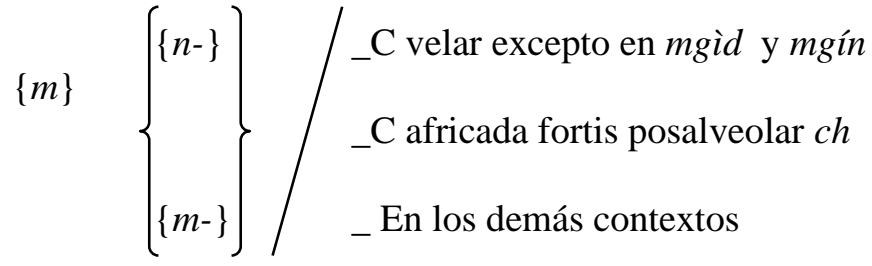

Regla 2. Alomorfía en los inicios de los nombres de animales

El alomorfo base para este sistema morfológico derivativo de nombres para animales es $\{m$ - $\}$. Este prefijo es el que ocurre en un mayor número de contextos. 


\section{Conclusiones}

En el ZSPM, existe un sistema morfológico derivativo utilizado para la formación de sustantivos. Este sistema se conforma de cuatro morfemas prefijales: $\{w-\},\{$ giel- $\},\{n g w-\}$ y $\{m-\}$. El prefijo derivativo nominalizador $w$ - se une a raíces verbales simples para derivar nombres de eventos. Este mismo morfema, al prefijarse a una raíz compleja formada de un verbo más un sustantivo, origina nombres abstractos. El segundo morfema es el nominalizador gièl-, que se adhiere a bases adjetivales, verbales y nominales para formar nominales con una semántica compleja. El tercer morfema es el nominalizador $n g w$ - que, al pegarse a verbos de acción, deriva nombres de agentes, mientras que al prefijarse a topónimos, origina gentilicios. El último prefijo derivativo de animacidad es $m$-, del cual resultan nombres de animales.

Este estudio, además de mostrar las estrategias sobre la morfología derivativa prefijal, también ofrece nuevas posibilidades de investigación, tales como la morfología derivativa sufijal, los cambios semánticos que ocurren al pasar de una categoría a otra, los cambios semánticos al pasar de una forma nominalizada simple a una forma nominalizada compleja, y de un estudio tipológico intragenético sobre la morfología derivativa en los idiomas zapotecos. Dichas temáticas se considerarán en futuras investigaciones.

\section{Referencias bibliográficas}

Antonio-Ramos, P. (2015). La fonología y morfología del zapoteco de San Pedro Mixtepec (Tesis doctoral inédita). Centro de Investigaciones y Estudios Superiores en Antropología Social, México.

Bauer, L. (2003). Introducing Linguistic Morphology (2. ${ }^{\text {a }}$ ed.). Washington, D. C., Estados Unidos: Georgetown University Press. 
Beam de Azcona, R. (2004). A Coatlan- Loxicha Zapotec Grammar (Unpublished doctoral dissertation). Universidad de California, Berkeley.

Beam de Azcona, R. (noviembre, 2018). Diversificación y convergencia: una reconstrucción del "zapoteco de la Sierra Sur” y la filiación del cisyautepequeño. Trabajo presentado en Seminario: Diacronía y sincronía en lenguas tipológicamente diversas: fundamentos diacrónicos en las explicaciones del cambio lingüístico de la Universidad de Sonora, Hermosillo, Sonora, México.

Bybee, J. (1985). A Study of the Relation between Meaning and Form. Amsterdam/Philadelphia: John Benjamins Publishing Company.

De Córdova, J. (1578). Vocabvlario en Lengva Çapoteca. México: INAH.

Genetti, C. (2014). How languages work: an introduction to language and linguistics. Cambridge, Inglaterra: Cambridge University Press.

Haspelmath, M. (2002). Understanding Morphology. New York, Estados Unidos: Oxford University Press.

Instituto Nacional de Estadística y Geografía. (2015). Censo de población y vivienda. México.

Kaufman, T. (2016). Proto-Sapotek(an) reconstructions. Institute for Mesaoamerican Studies, University at Albany, State University of New York. Recuperado de http://www.albany.edu/ims/PDLMA_publications_new.html

Malchukov, A., Haspelmath, M. y Bernard, C. (2010). Ditransitive constructions: a typology overview. En Andrej Malchukov, Martin Haspelmath y Bernard Comrie (Eds.), Studies in ditransitive constructions: a comparative handbook (pp. 1-64). Berlín, Alemania: Mouton de Gruyter. 
Marcus, J. y Kent F. (1978). Ethnoscience of the sixteenth-Century Valley Zapotec. En Richard I. Ford (Ed.), The Nature and Status of Ethnobotany. Ann Arbor, Michigan: 4 Anthropological Papers N. ${ }^{\circ} 67$.

Pickett, V., Black, C. y Cerqueda, V. M. (1998). Gramática popular del zapoteco del Istmo. Tucson, Arizona: Centro de Investigación y Desarrollo Binnizá.

Smith Stark, T. (2007). Algunas isoglosas zapotecas. En Cristina Buenrostro, Samuel Herrera Castro, Yolanda Lastra, Juan José Rendón, Otto Schumann, Leopoldo Valiñas y María Aydeé Vargas Monroy (Eds.), Clasificación de las lenguas indígenas de México. Memorias del III coloquio internacional de lingüística Mauricio Swadesh (pp. 69-133). México: Universidad Nacional Autónoma de México.

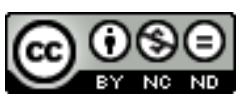

Esta obra está bajo una licencia de Creative Commons Reconocimiento-NoComercial-SinObraDerivada 4.0 Internacional 